\title{
Análise da volatilidade do Ibovespa entre 2001 e 2016: uma estimação por meio de modelos $\mathrm{ARCH}$
}

\author{
Volatility analisys of Bovespa Index from 2001 to 2016: an estimation using \\ ARCH models
}

Christian Ndege Kobunda e Enilson Carlos Nogueira Júnior*

\begin{abstract}
Resumo: O presente trabalho busca fazer uma análise da volatilidade do Índice Bovespa por meio de modelos simétricos e assimétricos da classe GARCH. Utilizando dados de 2001 a 2016, a modelagem da volatilidade mostra-se melhor ajustada pelos modelos GARCH $(1,1)$, EGARCH $(1,1)$ e TARCH $(1,1)$. Tais modelos confirmam o efeito persistência, visto que o somatório do coeficiente das variáveis em t-1 ser próximo a 1 . Em relação à assimetria, os modelos TARCH $(1,1)$ e EGARCH $(1,1)$ indicam que há diferença entre os impactos de choques positivos e negativos sobre a volatilidade, sendo que o primeiro também indica o "efeito alavacagem", ou seja, a volatilidade é maior em períodos de choques negativos.
\end{abstract}

Palavras-chave: Volatilidade; IBOVESPA; GARCH; Efeitos persistência; Alavancagem

Abstract: The article aims to analyze the volatility of the Bovespa Index through symmetric and asymmetric models of the GARCH class. Using data from 2001 to 2016, the volatility modeling is shown to be better adjusted by GARCH $(1,1)$, EGARCH $(1,1)$ and TARCH $(1,1)$ models. Such models confirm the persistence effect since the sum of the coefficient of the variables in $\mathrm{t}-1$ is close to 1 . Regarding the asymmetry, the TARCH $(1,1)$ and the EGARCH $(1,1)$ models indicate that there is a difference between impacts of positive and negative shocks on volatility. The first one also indicates the "leverage effect", which means that volatility is higher in periods of negative shocks.

Keywords: Volatility; IBOVESPA; GARCH; Persistence effect; Leverage effect

JEL Code: G10; C22

\footnotetext{
* Respectivamente, Mestre em Economia pela Universidade Federal de Espírito Santo (UFES). E-mail: christianndege@gmail.com; e Mestrando do Programa de Pós-Graduação em Economia Aplicada da ESALQ/USP. E-mail: enogueira@usp.br. DOI: http://dx.doi.org/10.5380/re.v40i72.63969
} 


\section{Introdução}

A necessidade de entender a volatilidade tornou-se mais urgente com a evolução da economia moderna. O grau de incerteza e os riscos endógenos e exógenos ao mercado, sobretudo financeiro, demandavam que as análises se debruçassem também sobre a heterocedasticidade condicional dos indicadores.

Os estudos de volatilidade em séries financeiras e o consequente uso dos modelos ARCH também se destacaram devido à necessidade de adequar a análise ao crescimento da importância do risco e das incertezas nas teorias econômicas modernas (BUENO, 2011).

Adicionalmente e buscando a análise para o objeto deste trabalho, importantes fatores, constantemente, sejam nacionais ou internacionais, têm afetado o desempenho da Bolsa de Valores de São Paulo, trazendo a discussão sobre os impactos no seu indicador, o Índice Ibovespa.

Nesse sentido, o estudo justifica-se pela necessidade de entender o comportamento estatístico do Ibovespa, sobretudo, em momentos de maior incerteza na economia local, propiciado, num primeiro momento, pela crise internacional em 2008/09 e, posteriormente, pela crise interna política e econômica no período pré-impeachment.

Adicionalmente, esse é um dos principais indicadores de representação do mercado acionário e financeiro nacional, e a volatilidade impacta diretamente na tomada de decisão de investidores internos e externos sobre os fluxos financeiros no país.

Nesse sentido, o presente trabalho tem os objetivos de (1) analisar o IBOVESPA, no período de 2001 a 2016, (2) sugerir e determinar os modelos mais indicados para a volatilidade do Índice e (3) analisar as características desses modelos, tais como, efeitos persistência, assimetrias e alavancagem.

$\mathrm{O}$ artigo está dividido em cinco seções. A primeira e segunda definem, teoricamente, a evolução dos modelos da classe GARCH, a saber, ARCH, GARCH, TARCH e EGARCH, e os procedimentos e práticas para as estimações de tais modelos. A terceira parte evidencia os principais trabalhos que buscam analisar a volatilidade do Ibovespa nos diversos períodos em questão. A quarta seção traz a análise do indicador, no período de 2001 a 2016, e apresenta, empiricamente, os modelos, metodologias e análises da volatilidade do mesmo. Por fim, lança as principais conclusões da análise, sugere passos para 
entendimento deste e alerta a importância do gerenciamento de risco para os agentes.

\section{Referencial teórico}

Os primeiros estudos sobre a heterocedasticidade condicional em modelos autorregressivos (classe ARCH) ocorreram a partir da década de 1980, em função da necessidade de se ter modelos financeiros e macroeconômicos que considerassem mais do que o primeiro momento condicional na série temporal. Bueno (2011) salienta que, em séries financeiras, a dependência temporal de momentos superiores era, até então, considerada como perturbações aleatórias e incondicionais, desconsiderando a existência de aglomerações (clusters) alternadas entre períodos de pequenas e grandes flutuações em torno da média, ou seja, a variância não era constante na série temporal.

Uma definição comum de volatilidade parte dessa característica, sendo considerada como a "variação, ao longo do tempo, da variância condicional de uma série temporal" (FILHO; FERNANDES; BAIDYA, 1993).

De acordo com Silva, Sáfadi e Júnior (2005), frequentemente o mercado financeiro apresenta período de intensa volatilidade, sobretudo em períodos de queda de preços, e tais choques tendem a gerar perturbações diferentes ao longo do tempo. Não por acaso, os indicadores financeiros se tornaram um terreno fértil para as análises de volatilidade e de aplicação dos modelos ARCH e dos modelos subsequentes como GARCH, EGARCH e TARCH.

No estudo pioneiro para a modelagem de volatilidade em séries temporais, Engle (1982) introduziu o modelo ARCH (autoregressive conditional heteroskedasticity), no qual a variância condicional dos erros (volatilidade) pode ser modelada pela defasagem do quadrado dos retornos distribuídos no passado.

Assim, o modelo ARCH (p) pode ser definido como:

$$
\begin{aligned}
& \epsilon_{t}=\sigma_{t} u_{t}, \quad u_{t} \sim \text { i.i.d } \\
& \sigma_{t}^{2}=\alpha_{0}+\alpha_{1} \epsilon_{t-1}^{2}+\ldots+\alpha_{p} \epsilon_{t-p}^{2}
\end{aligned}
$$

em que $\mathrm{p}$ determina o número de defasagens do retorno necessárias e o erro $\left(u_{t}\right)$ são as variáveis aleatórias com média igual a 0 e variância igual a 1 . Além disso, a estimação dos $\alpha_{p}$ ocorre por meio de uma regressão linear, de modo que $\alpha_{0}>0$ e $\alpha_{p} \geq 0$ e que $\sum_{i=1}^{p} \alpha_{i}<1$, o que garante o processo estacionário. Em termos de análise, o coeficiente $\alpha_{p}$ indica o tempo de reação, ou seja, o período 
necessário para que a volatilidade seja percebida na série.

Posteriormente, Bollerslev (1986), em resposta ao fato dos modelos ARCH (p) precisarem de muitos parâmetros para serem ajustados corretamente, sugeriu uma generalização do modelo $\mathrm{ARCH}$, o modelo GARCH (generalized autoregressive conditional heteroskedasticity).

No GARCH, a variância condicional do erro, além de ser explicada pela defasagem dos retornos no passado, deveria incluir a defasagem do quadrado da própria variância condicional em períodos anteriores. $\mathrm{O}$ autor advogava que $\mathrm{O}$ modelo era potencialmente mais parcimonioso que o anterior.

$\mathrm{O}$ modelo GARCH $(\mathrm{p}, \mathrm{q})$ pode ser resumido da seguinte maneira:

$$
\begin{gathered}
\epsilon_{t}=\sigma_{t} u_{t}, u_{t} \sim i . i . d, \\
\sigma_{t}^{2}=\alpha_{0}+\alpha_{1} \epsilon_{t-1}^{2}+\cdots+\alpha_{p} \epsilon_{t-p}+\beta_{1} \sigma_{t-1}^{2}+\cdots+\beta_{q} \sigma_{t-q}
\end{gathered}
$$

em que q é inserido para determinar o número de defasagens da própria variância condicional. Assume-se que o erro $\left(u_{t}\right)$ tenha as mesmas características da modelagem anterior e que $\alpha_{q} \geq 0$ e $\beta_{q} \geq 0$. Em termos de análise, o $\beta_{q}$ indica o quanto da volatilidade percebida do período anterior persiste no momento atual.

A estacionariedade é garantida se $\Sigma_{i=t} \alpha_{i}+\beta_{i}<1$, em que $\mathrm{n}=\operatorname{Max}$ $(\mathrm{p}, \mathrm{q})$.

Morettin e Toloi (2006) salienta que os modelos em que a heterocesdaticidade condicional, seja governada por modelos ARCH ou GARCH, supõe efeitos simétricos sobre os retornos, uma vez que é relacionada com o quadrado deles. No entanto, tal suposição não condiz com dados empíricos, nos quais, normalmente, choques negativos afetam os retornos em maior magnitude do que choques positivos, gerando efeitos assimétricos sobre o retorno (efeito alavancagem).

Nesse sentido, outras modelagens da classe GARCH surgiram posteriormente buscando capturar tais assimetrias das séries financeiras e, portanto, capturar o chamado "efeito alavancagem". Neste trabalho, ressaltamos as modelagens EGARCH e TARCH.

A primeira das modelagens citadas acima, o modelo de heterocedasticidade condicional autorregressivo generalizado exponencial, EGARCH, foi proposto por Nelson (1991) e insere a especificação logarítmica nos termos do modelo GARCH, permitindo a captura de efeitos assimétricos e a possibilidade de que alguns coeficientes sejam negativos, tendo em vista a especificação em log. 
Dessa forma, o EGARCH (p,q,r) modela a variância condicional como:

$$
\ln \sigma_{t}^{2}=\alpha_{0}+\Sigma_{\mathrm{t}=1}^{p} \sigma_{t}\left|\frac{\epsilon_{t-1}}{\sigma_{t-1}}\right|+\Sigma_{\mathrm{j}=1}^{q} \beta \ln \sigma_{t-j}^{2}+\Sigma_{\mathrm{k}=1}^{r} \gamma_{k} \frac{\epsilon_{\mathrm{t}-\mathrm{k}}}{\sigma_{\mathrm{t}-\mathrm{k}}}
$$

em que os coeficientes $\gamma_{k}$ ajustarão assimetria dos choques. Portanto, se $\gamma_{k}=0$, em todos os momentos $\mathrm{k}$, um choque positivo e negativo têm o mesmo efeito sobre a volatilidade, ou seja, os impactos são simétricos e não têm efeito alavancagem; se $\gamma_{k} \neq 0$, os impactos são assimétricos. Nesses casos, se $\gamma_{k}<0$, os choques negativos aumentam a volatilidade mais do que os choques positivos, $o$ que seria esperado em séries financeiras, como o Ibovespa.

A segunda modelagem também busca capturar a assimetria, o modelo de heterocedasticidade condicional generalizada com limitante, o TARCH ou Treshold ARCH, foi levantado por Zakoian (1994). Nessa modelagem, é inserida uma variável binária no modelo GARCH e os coeficientes têm que ser positivos para garantir a estacionariedade da série.

A definição de TARCH (p,q,r) pode ser resumida abaixo:

$$
\sigma_{t}^{2}=\alpha_{0}+\sum_{i=1}^{p} \alpha_{i} \epsilon_{t-i}^{2}+\sum_{j=1}^{q} \beta_{j} \sigma_{t-j}^{2}+\gamma_{i} d_{t-i} \epsilon_{t-i}^{2}
$$

em que d é a variável dummy que será igual a 1, se o erro satisfaz a condição imposta e, será 0 , se contrário. Isso quer dizer que, se houver uma notícia negativa, $\epsilon_{t-i}<0$, a dummy terá valor 1 e seu impacto será de $\alpha_{i}+\gamma_{i}$, caso contrário, não haverá efeito sobre a volatilidade. Além disso, se $\gamma_{k}>0$, há evidências de efeito alavancagem.

De modo geral, o modelo assume um crescimento da volatilidade quando há um choque negativo, $\epsilon_{t-k}<0$, uma vez que são acompanhadas por um coeficiente positivo $\left(\gamma_{k}>0\right)$, logo, sinalizando efeitos assimétricos sobre os retornos.

Os modelos acima são estimados por meio da Máxima Verossimilhança (condicional).

Ainda há outras extensões da classe GARCH, tais como o GARCH-M, PGARCH, entre outros, os quais não serão expostos aqui devido à quantidade e especificidades dos modelos e ao fato dos modelos acima já alcançarem o propósito do trabalho. 


\section{Procedimentos práticos para aplicação da metodologia}

Para o procedimento prática dos modelos especificados acima, primeiramente, define-se a volatilidade a partir do retorno diário do logaritmo da variável $(X)$. Logo, o retorno $\left(r_{t}\right)$ pode ser escrito como:

$$
r_{t}=\ln \left(X_{t}\right)-\ln \left(X_{t-1}\right)
$$

Após estimado a série de retornos, é necessário verificar a sua estacionariedade. Os testes de raiz unitária a serem realizados neste trabalho são propostos por Dickey e Fuller (1979) e Phillips e Perron (1988). O teste ADF (Aumented Dickey-Fuller) pode ser dado por:

$$
\Delta Y=\alpha+\beta_{t} t+\gamma Y_{t-1}+\sum_{i=1}^{m} \delta_{i} \Delta Y_{t-1}+\varepsilon_{t}
$$

em que $\Delta Y$ é operador de diferenças temporais de $\mathrm{Y}, \beta_{t}$ é o coeficiente de tendência do modelo, $\gamma$ é coeficiente da presença de raiz unitária, $\sum_{i=1}^{m} \delta_{i} \Delta Y_{t-1}$ são as defasagens incluídas no modelo e $\varepsilon_{t}$ é o termo aleatório.

O teste de Phillips-Perron considera a mesma regressão e é uma generalização do teste de Dickey-Fuller quando os erros são heterocedásticos. Em ambos os testes, a hipótese nula é de que a série é estacionaria, ou seja, não apresenta raiz unitária.

A fim de entender as dependências temporais presentes na série, é necessário fazer o uso de especificações de modelos ARMA, ou seja, um processo autorregressivo de médias móveis. Como a literatura destes é bastante vasta, esta seção se limitará a apresentação do modelo geral, o que será especificado diretamente ao IBOVESPA na seção de Resultados e Discussões. Um ARMA (p, q) pode ser escrito como:

$$
y_{t}=c+\sum_{i=1}^{p} \varphi_{i} y_{t-1}+\sum_{i=1}^{p} \theta_{j} \varepsilon_{t-1}
$$

em que $\varphi_{i}$ são os parâmetros associados à especificação autorregressiva $\left(y_{t-1}\right)$ e $\theta_{j}$ são os parâmetros associados às medias móveis dos erros no passado $\left(\varepsilon_{t-1}\right)$.

Para identificar e definir o modelo mais parcimonioso dentre os estimados, pode-se adotar a análise das Funções de Autocorrelação (FAC) e de Autocorrelação Parcial (FACP) e o uso de critérios de parcimônia como o Critério de Akaike (AIC), que pode ser definido como: 


$$
\operatorname{AIC}(p, q)=\ln \sigma_{p, q}^{2}+\frac{2(p+q)}{T},
$$

no qual $\sigma_{p, q}^{2}$ é o estimador de máxima verossimilhança de $\sigma^{2}$ e $\mathrm{T}$ as observações da série analisada.

Ainda existem outros critérios úteis e importantes na literatura como o Critério Bayesiano de Schwarz (SBC).

Antes de estimar os modelos da classe GARCH, é necessário observar a presença de heterocedasticia condicional autorregressiva nos resíduos do modelo. Para tal, Engle (1982) propôs o teste ARCH-LM para identificar se a covariância condicional é ou não constante na série. Considerando a regressão abaixo:

$$
\varepsilon_{t}^{2}=\alpha_{0}+\alpha_{1} \varepsilon_{t-1}^{2}+\cdots+\alpha_{p} \varepsilon_{t-s}^{2}+u_{t},
$$

pode-se definir um teste ARCH-LM em que

$$
T R^{2} \stackrel{d}{\rightarrow} \chi_{p}^{2}
$$

em que $R^{2}$ é o coeficiente de determinação da equação (3.5).

Se os coeficientes, em conjunto, forem próximos de zero, o R2 terá baixo poder explicativo. A hipótese nula apontada nesse teste é de que os resíduos da série são homocedásticos, isto quer dizer, não há a presença do modelo $\mathrm{ARCH}$ (BUENO, 2011).

\section{Referencial empírico}

Morais e Portugal (1999) iniciam as discussões sobre volatilidade na Bolsa de Valores de São Paulo, separando a análise em três períodos complicados da década de 1990, do ponto de vista internacional: crise do México, crise asiática e moratória russa.

Os autores utilizam modelos determinísticos e estocásticos e indicaram que os melhores modelos nos três períodos foram $\operatorname{GARCH}(1,1)$ e EGARCH $(1,1)$. Na análise entre os dois modelos, não houve diferença significa entre eles. Em termos de volatilidade, verificou-se, empiricamente, que períodos anteriores à crise efetivamente apresentavam volatilidade menor do que nos momentos das crises referidas acima.

Mota e Fernandes (2004) avaliaram o desempenho de diferentes métodos de extração da volatilidade da IBOVESPA tendo como referência a volatilidade encontrada no período de 01/08/1994 a 24/10/2001. Os autores propuseram 
análises de duas amostras - a primeira de 01/08/1994 a 03/04/1998 e a segunda de 06/04/1998 a 24/10/2001. Como resultado da comparação entre os modelos da classe GARCH e modelos alternativos, os autores encontraram que os estimadores alternativos são tão precisos quanto os modelos do tipo GARCH e menos trabalhosos em termos computacionais.

Posteriormente, Gaio et al. (2007) modelou os retornos do Índice de 2000 a 2005, encontrando a melhor especificação da volatilidade em EGARCH $(1,1)$, segundo o teste AIC e $\ln (\mathrm{L})$, sinalizando para presença do efeito alavancagem e para a persistência da volatilidade sobre a série no período. Adicionalmente, o autor ressalta que a mudança de governo em 2002 trouxe choques negativos sobre a volatilidade.

Em outro trabalho, Gaio e Sáfadi (2008) analisam a volatilidade dos retornos do Índice Bovespa, no período de 2000 a 2005, utilizando modelos de memória longa. Os autores encontraram que a série de retornos apresentava memória longa em sua volatilidade no período, caracterizando-se por FIEEGARCH $(1,1)$ com distribuição G.E.D. (Generalized error distribution) como o que melhor se ajusta à série, de ponto de vista preditivo.

Jubert et al. (2009) traz a discussão sobre as melhores formas de modelar o Ibovespa e seus índices setoriais pré-crise do subprime (2007-2008), analisando a volatilidade e seu padrão de simetria perante choques externos. $\mathrm{O}$ autor utiliza os modelos GARCH, TARCH e EGARCH, os quais evidenciaram o efeito alavancagem no período estudado nos índices setoriais e no Ibovespa. Todos os modelos foram mais bem ajustados para o movimento no dia anterior (t-1). Além disso, o autor compara as estimativas dos coeficientes entre os modelos simétricos (GARCH) e assimétricos (TARCH e EGARCH), indicando que, empiricamente, as estimativas são razoavelmente similares.

Especificamente sobre a crise do subprime, Righi e Ceretta (2013) estudaram o comportamento da volatilidade na última década e levantaram a hipótese de transmissão internacional de volatilidade sobre o Ibovespa. Utilizando um GARCH bivariado entre o mercado americano, argentino, mexicano e chinês, os autores chamam atenção para mudança no sinal da direção da transmissão, com o Brasil tendo maior influência sobre os outros mercados, e ressalta que a relação é menos assimétrica que anteriormente a crise. A hipótese levantada pelo trabalho é de que isso é resultado da maior resiliência de curto prazo que o mercado 
brasileiro observou no período pós-crise, diante das medidas anticíclicas tomadas pelo governo.

Outra análise relevante para explicar a volatilidade de dois dos principais papéis inseridos no IBOVESPA foi a que Da Silva (2009) fez ao examinar o processo do retorno das ações preferenciais da Petrobras e da Vale entre 03/06/2000 e 11/08/2008, testando as reações de persistências e assimetrias na volatilidade dos retornos. Utilizando modelos heteroscedásticos, o autor encontrou evidências de que os choques negativos e positivos têm impactos diferenciados sobre a volatilidade dos retornos. Comprovado por meio de modelo EGARCH $(1,1)$ e TARCH $(1,1)$. E, por fim, com base no critério de raiz do erro quadrático médio (REQM), o autor escolheu EGARCH $(1,1)$ como melhor modelo de previsão da volatilidade das ações da Petrobras e da Vale.

De modo geral, em termos metodológicos, prevalecem nos trabalhos anteriores os modelos de ordem baixa e assimétricos, apesar de algumas evidências que as estimativas entre os modelos simétricos e assimétricos são parecidas. Em termos analíticos, percebe-se que eventos externos, como crises internacionais, normalmente apresentam impactos diretos sobre a volatilidade do Índice, o que é esperado diante de um mercado financeiro nacional aberto.

\section{Resultados e discussão}

O Índice Bovespa ou IBOVESPA é um indicador representativo de uma carteira teórica das ações mais negociadas na Bolsa e, de modo geral, consegue captar o comportamento médio das cotações diárias na Bovespa. Os dados do Índice Bovespa foram coletados no próprio site do BMF Bovespa, com recorte temporal de 02/01/2001 a 30/12/2016, ( $n=3992)$, e periodicidade diária.

A análise gráfica mostra períodos de maior variabilidade na época da crise do subprime, em 2008 e 2009, diante das incertezas no cenário internacionais ${ }^{\dagger}$. No restante da série, observa-se alguns curtos períodos de maior variabilidade, no entanto, de menor magnitude, se comparados com o período analisado anteriormente. †Nesse sentido, Chaine (2011) levanta e confirma a hipótese de contágio entre os mercados financeiros do
Brasil e dos EUA. 
Figura 1 - Índice Bovespa (2001-2016)

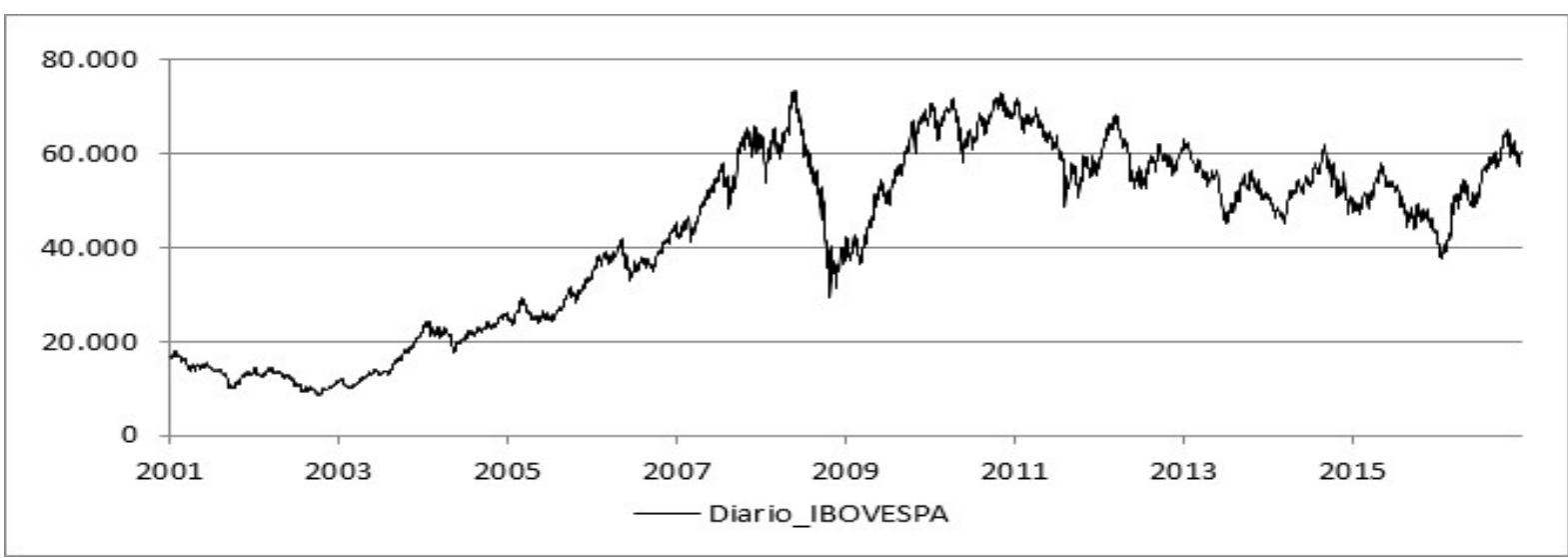

Fonte: BMF Bovespa. Elaboração a partir de dados do R Studio.

Figura 2 - Retornos diários (log_retorno) do Índice Bovespa (2001-2016)

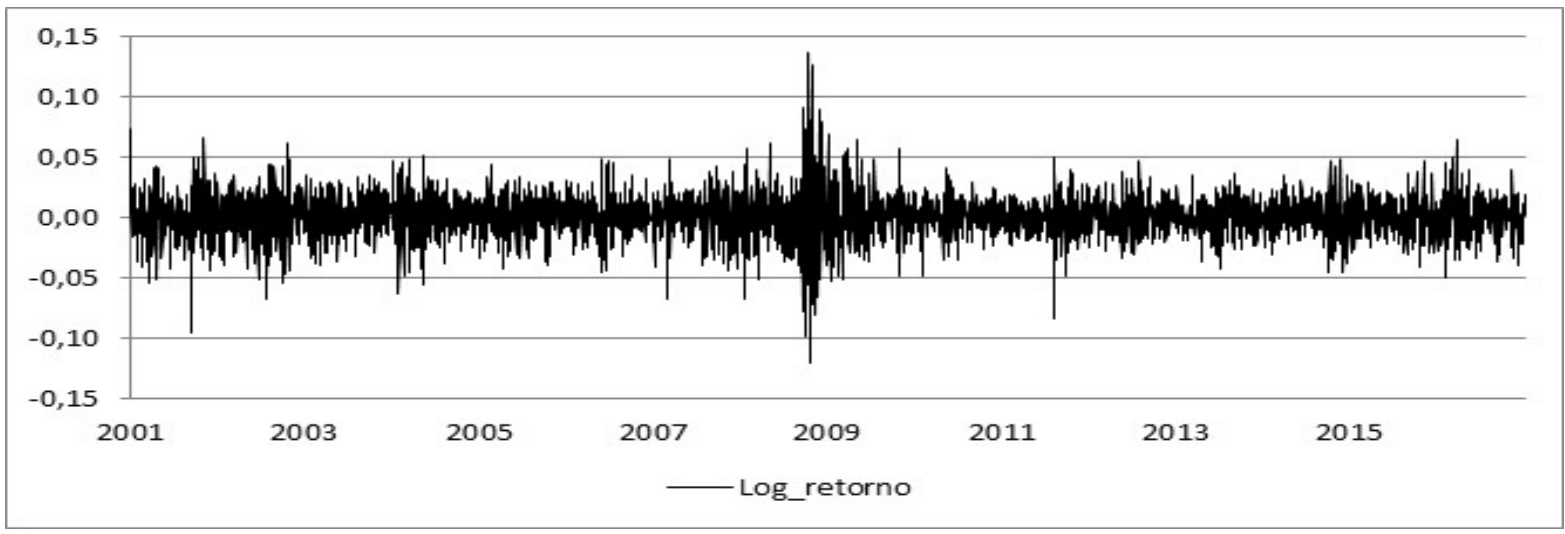

Fonte: BMF Bovespa. Elaboração a partir de dados do R Studio.

Na Tabela 1, o valor estimado acima de 3 para o coeficiente de curtose aponta para a característica leptocúrtica dos retornos diários da Ibovespa (dispersão afunilada em relação à distribuição normal). A leptocurtose também pode ser observada no histograma (Figura 3).

Além disso, o teste de normalidade dos resíduos proposto por Jarque e Bera (1987) sugere que a série não apresenta distribuição normal, uma vez que o P-valor do teste é menor que $1 \%$. Portanto, o excesso de curtose e a consequente rejeição da normalidade na série já eram esperadas numa série financeira como o Ibovespa, uma vez que indicam para alguns dos fatos estilizados comuns nessas séries. 
Tabela 1 - Estatísticas descritivas para IBOVESPA e para os retornos diários

\begin{tabular}{lcc}
\hline Estatistica & IBOVESPA & Retornos diários \\
\hline Média & 42.927 & 0,0003 \\
Mediana & 49.151 & 0,0005 \\
Máximo & 73.516 & 0,1368 \\
Mínimo & 8.370 & $-0,121$ \\
Desvio padrão incondicional & 18.861 & 0,018 \\
Assimetria & $-0,4276$ & $-0,0966$ \\
Coeficiente de curtose & $-1,1782$ & 4,0649 \\
JarqueBera & 352,3 & $2.758,70$ \\
p-valor & $2,2, \mathrm{E}-16$ & $2,2, \mathrm{E}-16$ \\
\hline
\end{tabular}

Fonte: dados da pesquisa. Elaboração a partir de dados do R Studio.

\section{Figura 3 - Histograma da distribuição dos retornos diários (log_retorno)}

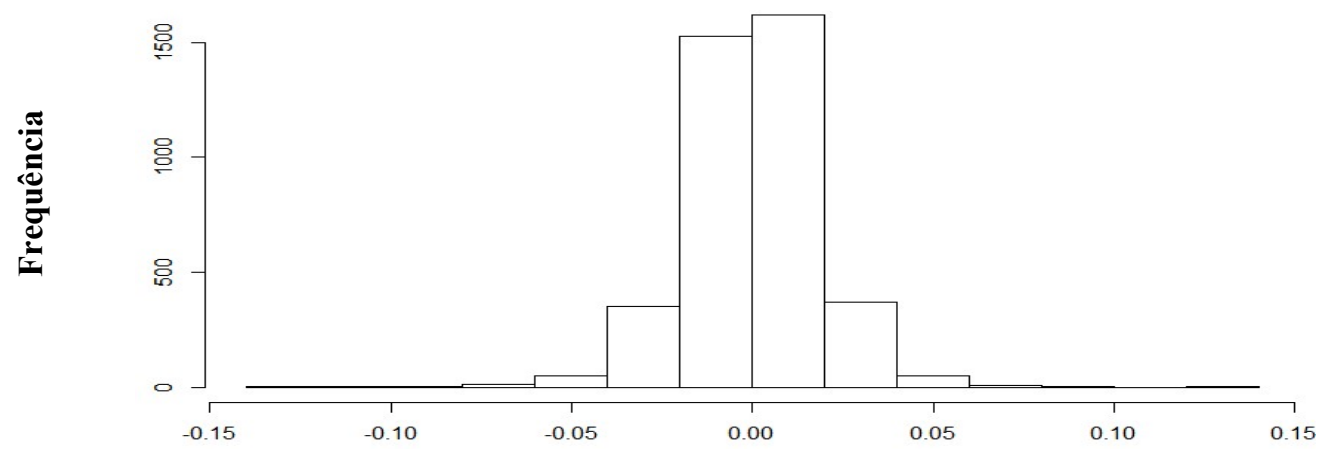

Fonte: dados da pesquisa. Elaboração a partir de dados do R Studio.

Prosseguindo, vale ressaltar que os retornos diários foram definidos como o logaritmo natural da variação diária do IBOVESPA, conforme Morais e Portugal (1999) e Gaio et al. (2007), por isso, o termo log retorno. Além disso, tal indicador permite analisar a variação do indicador e não seu patamar, desta forma, normalmente essa série é estacionária.

Tal afirmativa também se mostra verdadeira para a série analisada, conforme descrito na Tabela 2. Por meio dos testes propostos por Dickey e Fuller (1979) e Phillips e Perron (1988), a série de retornos do IBOVESPA não apresenta raiz unitária, uma vez que nos dois testes há a rejeição da hipótese alternativa (não estacionariedade) para os valores a 1\%, 5\% e 10\% de significância. 
Tabela 2 - Testes de estacionariedade do retorno do IBOVESPA

\begin{tabular}{lc}
\hline Teste & Estatísticas T - Retornos diários \\
\hline Philips-Perron & $-63,176$ \\
Dickey-Fuller Aumentado & $-62,975$ \\
\hline
\end{tabular}

Fonte: dados da pesquisa. Elaboração a partir de dados do RStudio.

Valores críticos para os testes: $1 \%,-2,58 ; 5 \%,-1,95 ; 10 \%,-1,62$.

Com a série estacionária, buscou-se determinar empiricamente o melhor modelo autorregressivo e de média móvel para a série. Assim como o trabalho de Gaio et al. (2007), ajustou-se o modelo AR (1). Esse modelo também se apresenta como o melhor de acordo com o Critério de Akaike (AIC).

Como uma forma alternativa, as Funções de Autocorrelação (FAC) e de Autocorrelação Parcial (FACP) também indicam para a espeficicação AR (1).

\section{Figura 4 - FAC e FACP para a variável retornos diários (log_retorno)}
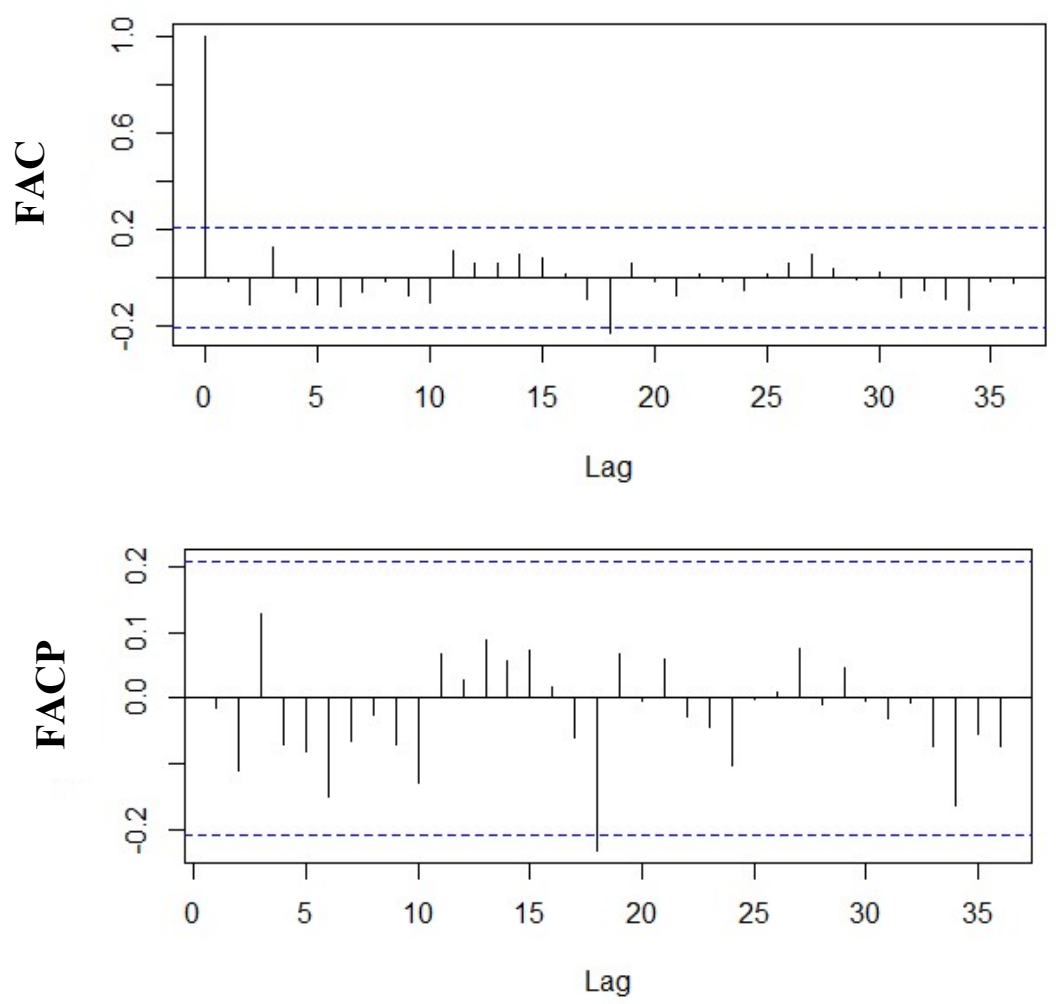

Fonte: dados da pesquisa. Elaboração a partir de dados do R Studio.

O teste ARCH-LM foi proposto por Engle (1982), com intuito de identificar a presença de efeitos ARCH numa série de resíduos do modelo autorregressivo. A hipótese nula apontada nesse teste é de que os resíduos da série são homocedástico, 
ou seja, não apresentam efeitos ARCH.

Como pode ser visto na Tabela 3, para todas defasagens estimadas, o teste ARCH-LM sugere a rejeição da hipótese nula para os resíduos de AR (1) à 1\% e, logo, há efeitos ARCH sobre tal série de resíduos do modelo autorregressivo de primeira ordem.

Tabela 3 - Testes ARCH-LM sobre os resíduos

\begin{tabular}{lcc}
\hline \multicolumn{1}{c}{ Teste } & Estatística & valor-p \\
\hline Lags1 & 91,2 & $2,2, \mathrm{E}-16$ \\
Lags5 & 717,1 & $2,2, \mathrm{E}-16$ \\
Lags 10 & 954,8 & $2,2, \mathrm{E}-16$ \\
\hline
\end{tabular}

Fonte: dados da pesquisa. Elaboração a partir de dados do R Studio.

Após a confirmação da heterocesdaticidade condicional no modelo, foram estimados vários modelos da classe GARCH para identificação dos modelos estatisticamente significativos, considerando a teoria apresentada no segundo capítulo e os outros trabalhos do Ibovespa.

Além disso, é valido indicar que, segundo Morettin e Toloi (2006), a identificação da ordem de uma modelo da classe GARCH normalmente é difícil, sendo recomendado que se utilize modelos de baixa ordem. Seguindo isso e selecionando as especificações estatisticamente significantes dos modelos, temos os resultados da Tabela 4 .

Na Tabela 4, primeiramente, todos os parâmetros dos modelos selecionados são estatisticamente significativos ao nível de 1\%, possibilitando uma boa estimativa nos modelos e uma análise consistente da volatilidade do Ibovespa e dos impactos de choques externos.

Observa-se que os modelos mais indicados são de ordem 1, o que seria esperado, haja vista os trabalhos levantados na terceira seção deste artigo. Esse fato aponta que o entendimento da volatilidade do IBOVESPA depende somente do momento anterior (t-1). Além disso, percebe-se que os coeficientes são relativamente parecidos nos modelos simétricos e assimétricos.

Em relação ao modelo simétrico, os coeficientes do modelo GARCH $(1,1)$ sugerem que há uma considerável persistência de choques na volatilidade e do retorno passado sobre os períodos posteriores, uma vez que o somatório dos parâmetros $(\sim 0,98)$ fica muito próximo a 1 . Nesse sentido, admite-se que $92 \%$ da volatilidade do dia anterior permanece no dia seguinte. 
Tabela 4 - Resultados da estimação dos modelos da classe ARCH

\begin{tabular}{ccccc}
\hline Var. condicional & Variável & GARCH (1,1) & EGARCH (1,1) & TGARCH (1,1) \\
\hline$\alpha_{0}$ & & $0,0001^{*}$ & $-0,1533^{*}$ & $0,0001^{*}$ \\
$\alpha_{1}$ & $\epsilon_{t-1}^{2}$ & $(-3820)$ & $(-102,2)$ & $(-4088)$ \\
$\beta_{1}$ & $\sigma_{t-1}^{2}$ & $(-76074)$ & & $0,0460^{*}$ \\
$\alpha_{1}$ & $\left|\frac{\epsilon_{t-1}}{\sigma_{t-1}}\right|$ & $\left(-9236^{*}\right.$ & & $(-4267)$ \\
$\beta_{1}$ & $\ln \sigma_{t-1}^{2}$ & & $-0,9264^{*}$ \\
$\gamma_{1}$ & $\frac{\epsilon_{\mathrm{t}-1}}{\sigma_{\mathrm{t}-1}}$ & & $(-8,229)$ & $(-86545)$ \\
$\gamma_{1}$ & $d_{\epsilon_{t-k}}<0 \epsilon_{t-k}$ & $(-3076)$ & \\
\hline
\end{tabular}

Fonte: dados da pesquisa. Elaboração a partir de dados do R Studio.

Estatística $t$ entre parênteses. * Significância estatística a 1\%, 5\% e $10 \%$.

Aprofundando a análise, nota-se a presença do efeito alavancagem nos dois modelos assimétricos propostos. Primeiramente, o coeficiente $\gamma 1$ do modelo EARCH $(1,1)$ mostra-se estatisticamente consistente a $1 \%$, ou seja, há efeitos assimétricos em choque positivos e negativos. No entanto, por ser positivo, não sugere efeito alavancagem.

Aqui cabe uma discussão do valor esperado para o coeficiente acima. $\mathrm{O}$ valor esperado de $\gamma 1$ seria negativo, sugerindo que os choques negativos são acompanhados de volatilidades mais altas.

Nos parâmetros estimados no modelo TARCH $(1,1)$, o coeficiente é estatisticamente diferente de 0 e positivo. Isso também sugere que, além da assimetria, choques negativos geram período de volatilidade mais forte que choques positivos, portanto, sugerindo o efeito alavancagem sobre a série nesse período.

Os últimos dois modelos analisados convergem para característica assimétrica da volatilidade, no entanto divergem em relação ao efeito alavancagem. Nas propriedades de cada modelo, nota-se uma vantagem do modelo EGARCH, dado que pode haver coeficientes negativos e é, justamente, o sinal de um desses coeficientes que apontam para o efeito alavancagem. Ressalta-se também que o sinal do coeficiente $\gamma 1$ no modelo TARCH e EGARCH são diferentes para indicar o efeito alavancagem, uma vez que, no modelo TARCH, o 
sinal deveria ser positivo, enquanto que, no EGARCH, o sinal esperado é negativo. A análise da construção do modelo ajuda a explicar a oposição entre os modelos no que diz respeito à alavancagem.

O presente trabalho nos permite sugerir que há uma elevada persistência das variáveis no passado sobre a atual volatilidade, tendo em vista os altos coeficientes estimados nos 3 modelos, sobretudo para a volatilidade no passado. Ademais, os modelos convergem para assimetria, contudo somente o TARCH $(1,1)$ sugere para efeito alavancagem, o que seria esperado numa série financeira.

Tabela 5 - Critérios de informação dos modelos selecionados

\begin{tabular}{lcccc}
\hline \multicolumn{1}{c}{ Modelo } & Akaike & Baysiano & Shibata & Hannan-Quin \\
\hline GARCH $(1,1)$ & $-5,39997$ & $-5,39367$ & $-5,39997$ & $-5,39774$ \\
EGARCH $(1,1)$ & $-5,40860$ & $-5,39750$ & $-5,40860$ & $-5,40470$ \\
TARCH $(1,1)$ & $-5,41190^{*}$ & $-5,40086^{*}$ & $-5,41191^{*}$ & $-5,40799^{*}$ \\
\hline
\end{tabular}

Fonte: dados da pesquisa. Elaboração a partir de dados do R Studio.

* representa o melhor modelo segundo cada critério.

Segundo os critérios de informação analisados, o modelo com melhor ajuste é o TARCH $(1,1)$, uma vez que este apresenta os menores valores de todos dos critérios (Akaike, Baysiano, Shibata e Hannan-Quin). Isso sugere para a efetividade do efeito alavacagem na série do Ibovespa.

\section{Conclusão}

Por meio da proposta de modelagem da volatilidade do Índice Bovespa de 2001 a 2016, o presente trabalho levanta argumentos para análises do tal índice dentro das características de uma série financeira. O primeiro deles se dá na análise descritiva do Ibovespa e do seu retorno, na forma logarítmica. Percebem-se, nesse sentido, a característica assimétrica e leptocúrtica e a rejeição da normalidade dos erros dos indicadores listados acima.

$\mathrm{Na}$ modelagem da volatilidade, nota-se que os modelos simétricos e assimétricos apresentam parâmetros razoavelmente semelhantes e de baixa ordem. Os modelos propostos, GARCH $(1,1)$, EGARCH $(1,1)$ e TARCH $(1,1)$, convergem para a presença de persistência da volatilidade passada em momentos posterior e para a presença de assimetria entre choques positivos e negativos, evidenciando que choques negativos são acompanhados de maiores períodos de volatilidade do que choques positivos. Visto que a volatilidade no mercado é susceptível a reações 
de persistência e assimetria, sugere-se que o índice tenha característica de memória longa, ou seja, os impactos, positivos ou negativos, são notados ao longo do tempo.

Por fim, entende-se que, considerando tal persistência e assimetria, é sugerido que os agentes, públicos e privados, tomem ações para melhor gerenciamento dos riscos exógenos ao mercado acionário, com intuito de mitigar tais fatores e diminuir a exposição à volatilidade, sobretudo, quando houver choques negativos sobre o índice.

\section{Referências}

BUENO, R. L da S. Econometria de Séries Temporais. São Paulo: Cengage Learning, 2011.

BOLLERSLEV, T. Generalized autoregressive conditional heteroskedasticity. Journal of Econometrics, v. 31, n. 3, p. 307-327, 1986.

BOLLERSLEV, T.; ENGLE, R. F.; WOOLDRIDGE, J. M. A capital asset pricing model with time-varying covariances. Journal of Political Economy, v. 96, n. 1, p. 116-131, 1988.

CHAINE, M. Testando a hipótese de contágio entre o Índice Bovespa e o S\&P500 ao longo da crise de 2008 com modelos multivariados de volatilidade. Revista de Economia e Administração, v. 10, n. 2, 2011.

DA SILVA, C. A. G. Modelagem de estimação da volatilidade do retorno das ações brasileiras: os casos da Petrobrás e Vale. Cadernos do IME-Série Estatística, v. 26, n. 1, p. 15, 2009.

DICKEY, D. A.; FULLER, W. A. Distribution of the estimators for autoregressive time series with a unit root. Journal of the American Statistical Association, v. 74, n. 366a, p. 427-431, 1979.

DUARTE, A.; HEIL, T.; PINHEIRO, M. Estimação da volatilidade de ativos e índices brasileiros. Resenha $B M \& F$, v. 111, 1996.

ENGLE, R. F. Autoregressive conditional heteroscedasticity with estimates of the variance of United Kingdom inflation. Econometrica: Journal of the Econometric Society, p. 987-1007, 1982.

FILHO, A. L. F.; FERNANDES, CAC; BAIDYA, T. Medidas de Volatilidade para Opções. XXV sbpo/sobrapo, n. 1, p. 185-187, 1993. 
GAIO, L. E.; PESSANHA, G. R. G.; OLIVEIRA, D. R.; ÁZARA, L. N. Análise da volatilidade do índice Bovespa: um estudo empírico utilizando modelos da classe ARCH. Contextus-Revista Contemporânea de Economia e Gestão, v. 5, n. $1,2007$.

GAIO, L. E.; SÁFADI, T. Memória longa na volatilidade do Índice BOVESPA: uma análise utilizando modelos da classe ARCH. Revista de Economia $e$ Administração, v. 7, n. 2, 2008.

JARQUE, C. M.; BERA, A. K. A test for normality of observations and regression residuals. International Statistical Review/Revue Internationale de Statistique, p. 163-172, 1987.

JUBERT, R. W.; MONTE, P. A.; PAIXÃO, M. C. S.; LIMA, W. H. Um estudo do padrão de volatilidade dos principais índices financeiros do Bovespa: uma aplicação de modelos ARCH. Revista Contabilidade, Gestão e Governança, v. 11, n. 1-2, 2009.

MORAIS, I. A. C.; PORTUGAL, M. S. Modelagem e previsão de volatilidade determinística e estocástica para a série do Ibovespa. 1999.

MORETTIN, P. A.; TOLOI, C. Análise de séries temporais. ABE - Projeto Fisher. Editora Blucher, 2006.

MOTA, B. S.; FERNANDES, M. Desempenho de estimadores de volatilidade na bolsa de valores de São Paulo. Revista Brasileira de Economia, v. 58, n. 3, p. 429448, 2004.

PHILLIPS, P. C. B.; PERRON, P. Testing for a unit root in time series regression. Biometrika, v. 75, n. 2, p. 335-346, 1988.

NELSON, D. B. Conditional heteroskedasticity in assetreturns: A new approach. Econometrica: Journal of the Econometric Society, p. 347-370, 1991.

RIGHI, M. B.; CERETTA, P. S. Efeito da crise de 2007/2008 na transmissão internacional de volatilidade no mercado de capitais brasileiro. REAd-Revista Eletrônica de Administração, v. 19, n. 2, p. 384-400, 2013.

SILVA, W. S.; SÁFADI, T.; CASTRO JÚNIOR, L. G. Uma análise empírica da volatilidade do retorno de commodities agrícolas utilizando modelos ARCH: os casos do café e da soja. Revista de Economia e Sociologia Rural, v. 43, n. 1, p. 119-134, 2005.

ZAKOIAN, J. Threshold heteroskedastic models. Journal of Economic Dynamics andcontrol, v. 18, n. 5, p. 931-955, 1994. 